\title{
Control of Hypertension among Diabetic Patients in a Referral Hospital in Tanzania: A Cross-Sectional Study
}

\author{
Semvua B. Kilonzo,2*, Daniel W. Gunda ${ }^{1,2}$, Fatma A. Bakshi ${ }^{1,2}$, \\ Fredrick Kalokola $^{1,2}$, Henry A. Mayala ${ }^{4}$, Hollo Dadi ${ }^{3}$
}

\section{OPEN ACCESS}

Citation: Semvua B. Kilonzo, Daniel W. Gunda, Fatma A. Bakshi, Fredrick Kalokola, Henry A. Mayala, Hollo Dadi.Control of Hypertension among Diabetic Patients in a Referral Hospital in Tanzania: A Cross-Sectional Study. J Health Sci 2017;27(5):473. doi:http://dx.doi.org/10.4314/ejhs.v27i5.5

Received: February 14, 2017

Accepted: April 23, 2017

Published: September 1, 2017

Copyright: (c) 2017 Semvua B. K, et al.

This is an open access article distributed under the terms of the Creative Commons

Attribution License, which permits unrestricted use, distribution, and reproduction in any medium, provided the original author and source are credited.

Funding: Nil

Competing Interests: The authors declare that this manuscript was approved by all authors in its form and that no competing interest exists.

Affiliation and Correspondence:

${ }^{1}$ Department of Internal Medicine,

Catholic University of Health and

Allied Sciences, Mwanza-Tanzania

${ }^{2}$ Department of Internal Medicine,

Bugando Medical Centre,Mwanza-

Tanzania

${ }^{3}$ Faculty of Undergraduate Studies,

Weill School of Medicine, Mwanza-

Tanzania ${ }^{4}$ Cardiovascular department,

Jakaya Kikwete Cardiac Institute,

Dar es Salaam-Tanzania

"Email: sekipcb@yahoo.com

\section{ABSTRACT}

BACKGROUND: Hypertension is common among diabetic patients. The co-existence of two conditions carries an excessive risk of severe complications and mortalities. Limited information exists on the determinants of poor hypertension control among these patients. We aimed at determining the prevalence and factors associated with poor hypertension control in these patients.

METHODS: Data of diabetic patients who were also hypertensive attending an outpatient clinic from 1 August 2015 to 31 December 2015 at Bugando Medical Centre were retrospectively analyzed. Uncontrolled hypertension was defined as a blood pressure of $\geq 130 \mathrm{mmHg}$ and/or $\geq 80 \mathrm{mmHg}$ systolic and diastolic respectively. $A$ designed questionnaire was used to collect data of patients. Continuous variables were summarized by median and interquartile ranges (IQR) and categorical variables were summarized by frequency and percentage. Logistic regression was used to find the predictors of uncontrolled hypertension.

RESULTS: The majority of our study population were females, 161/295 (54.6\%), and the median age was 57 years (IQR 50-64). The prevalence of hypertension was $206 / 295$ (69.8\%). A total of 174/206 (84.5\%) patients had uncontrolled hypertension. This poor control was significantly associated with poor adherence to antihypertensives (OR 1.73[1.26-2.38] $p=0.002)$, presence of any longterm complication (OR 3.19 [1.65-6.18] $p=0.03$ ) and overweight (BMI>24.9 Vs <24.9) (OR 1.68 [0.98-2.88], $p=0.04)$. Underprescription and ambiguous drugs combination was also observed. CONCLUSION: The prevalence of poor hypertension control among diabetic patients in Tanzania is alarming. Most of the factors associated with this situation can be modified. The clinicians should advocate individualized management, continuous health education and adherence to the available guidelines. KEYWORDS: Uncontrolled hypertension diabetes Tanzania 


\section{INTRODUCTION}

There is a growing evidence that the burden of non-communicable diseases (NCDs) is rapidly increasing in sub-Saharan Africa (SSA) (1-3). Estimates of age-standardized mortality suggest that patients in SSA may have up to three-fold higher mortality rates than similar European cohorts for non-communicable diseases. Diabetes mellitus (DM) and hypertension are common diseases in Africa with high mortalities (4). Hypertension, a common comorbid in diabetes, affects more than $50 \%$ of diabetic patients (5-7). It is clear that the co-existence of these two conditions in a patient carries an excessive risk for both micro- and macro-vascular complications, and mortalities (8). A Framingham study, one of the largest meta-analysis surveys, showed a strong association between the two diseases. Coexistence of hypertension in diabetics is attributed to the risk of death and cardiovascular events by $44 \%$ and $41 \%$ respectively as compared to $7 \%$ and $9 \%$ risk by diabetes alone (9).

Blood pressure lowering in diabetic patients has a remarkable cardiovascular $(\mathrm{CV})$ protective effect. Guidelines from the Joint National Committee (JNC-7) and American Diabetes Association (ADA) recommend maintaining blood pressure at $<130 \mathrm{mmHg}$ systolic and $<80 \mathrm{mmHg}$ diastolic in patients with diabetes. Usually, multiple anti-hypertensive agents are needed to achieve this goal $(8,10)$. In most cases, adequate control of blood pressure (BP) in these patients is not attained $(5,7,11)$. Information on the prevalence, control and risk factors of poor control of hypertension among diabetic patients in Tanzania is scarce. The objectives of this study were to determine the prevalence of hypertension among diabetics, to evaluate the degree of $\mathrm{BP}$ control, to describe the factors associated with poor BP control, and to evaluate the patterns of antihypertensive treatment prescription.

\section{MATERIALS AND METHODS}

This cross-sectional study was conducted in a diabetic clinic at Bugando Medical Centre (BMC). $\mathrm{BMC}$ is the zonal hospital for the Lake Victoria region in Northwest Tanzania, and it serves a population of approximately 13 million people. The clinic follows around 1000 diabetic patients from Mwanza region and referrals from around the Lake Zone. This study was conducted over a period of four months starting from August 2015.

Study population: We retrospectively reviewed clinical records of all patients who were attending our diabetic clinic from 01 August to 31 December 2015. Patients were excluded if they were younger than 18 years or pregnant. Patients' age, gender, occupation, smoking history, duration of diabetes, history of hypertension, use of antihypertensive and/or hypoglycemic drugs were recorded on structured questionnaires. Smoking history in this study was defined as being either previous or current smoker. Poor adherence to medications was defined as a patient's selfreported use of $<80 \%$ of the prescribed drugs. This cut-off is widely acceptable and has been used in other studies for chronic drugs adherence (12). Other related clinical information was also documented. This included height, weight, blood pressures, Fasting Blood Glucose (FBG), cholesterol levels, presence of any documented chronic complication(s) and use of other long term medications. Body Mass Index (BMI) was calculated as $\mathrm{kg} / \mathrm{m}^{2}$ using the most recent records. The mean blood pressure from three recent visits was used to assess the degree of blood pressure control. Controlled hypertension was defined as the mean BP of $<130 / 80 \mathrm{mmHg}$. FBG measurements were also recorded. The mean FBG from three recent visits was used to define the Glycemic control. Patients were labeled to have poor glycemic control when average FBG was $>7.0 \mathrm{mmol} / \mathrm{L}$. Glycosylated hemoglobin (HbA1C) was not done in these patients. The mean FBG of 7.0Mmol/L, which has been used as a cut-off point in this study, is justified by its correlation to around $6 \%$ HbA1C (13). The mean total cholesterol level from two recent consecutive visits was used to define the total cholesterol in this study.

Ethical statement: The study was approved by Bugando Medical Centre/ Catholic University of Health and Allied Sciences (BMC/CUHAS) joint Committee of Research and Publications.

DOI: http://dx.doi.org/10.4314/ejhs.v27i5.5 
Data analysis: Data was entered, verified and cleaned using Microsoft excel and analysis was done using STATA version 14 (College Station, Texas). Continuous variables were summarized by medians and interquartile ranges (IQRs), and categorical variables were summarized by frequency and percentage. Logistic regression was used to find the predictors of poor blood pressure control. Comparisons between the two groups were computed using chi-squared test for categorical variables, nonparametric Wilcoxon rank-sum test for continuous variables and student t-test to compare means between the groups.
Baseline characteristics of 295 enrolled patients attending diabetic clinic at BMC: A total of 295 patients attending diabetic clinic at the BMC from August to December 2015 fulfilled the inclusion criteria. Among these patients, 161/295 (54.6\%) were females. The median age was 57 years (IQR 50-64). The mean Body Mass Index (BMI) of our patients was high $27 \mathrm{~kg} / \mathrm{m}^{2}$ (IQR 24-30). The mean duration of diabetes was 48-months (IQR 22-120), and hypoglycemic (anti-diabetic) drugs were prescribed to all patients. An average FBG in three consecutive visits was 9.6 (6.9-13.6) $\mathrm{Mmol} / \mathrm{dL}$ (Table 1).

\section{RESULTS}

Table 1: Baseline characteristics of 295 enrolled diabetic patients attending diabetic clinic at BMC

\begin{tabular}{lll}
\hline Characteristic & & Number (\%) or median (IQR) \\
\hline Sex & Male & $134(45.4)$ \\
& Female & $161(54.6)$ \\
Age in years & & $57(50-64)$ \\
BMI $\left(\mathrm{kg} / \mathrm{m}^{2}\right)$ & Single & $27(24-30)$ \\
Marital status & Married & $5(1.78)$ \\
& Divorced & $79(27.9)$ \\
& Widowed & $145(51.2)$ \\
Address & Mwanza city & $54(19.0)$ \\
& Outside of Mwanza city & $103(34.9)$ \\
Work status & Informal employment & $196(65.1)$ \\
& Formal employment & $76(25.9)$ \\
Smoking history & Retired & $22(7.5)$ \\
& Yes & $32(10.9)$ \\
Duration of DM (months) & No & $262(89.1)$ \\
Duration on Anti-diabetics (Months) & & $48(22-120)$ \\
Average FBG (Mmol/dl) (3 consequent visits) & & $36(14-84)$ \\
Hypertensive & Yes & $9.6(6.9-13.6)$ \\
Possession of any health insurance & No & $206(69.8)$ \\
& Yes & $89(43.2)$ \\
& No & $91(31.2)$ \\
& & $201(68.8)$ \\
\hline
\end{tabular}

Prevalence of blood pressure control among diabetic patients attending diabetic clinic at BMC: Out of 295 diabetic patients attending diabetic clinic, 206(69.8\%) were hypertensive. The median systolic and diastolic blood pressures were $140(120-150) \mathrm{mmHg}$ and $84(78-89) \mathrm{mmHg}$ respectively. A total of 174/206 (84.5\%) patients had uncontrolled BP $(\geq 130 / 80 \mathrm{mmHg})$.

Characteristics of 206 diabetic patients with hypertension attending diabetic clinic at BMC: Table 2 displays the baseline and clinical characteristics of hypertensive-diabetic patients. Fifty-six percent of these patients were females.

DOI: http://dx.doi.org/10.4314/ejhs.v27i5.5 
Many hypertensive patients, 117 (56.8\%), were in the age group of 51-65 years. Severe hypertension was seen in 57/206(27.7\%) and 40/206(19.4\%) patients for systolic BP $\geq 160 \mathrm{mmHg}$ and diastolic $\geq 100 \mathrm{mmHg}$ respectively. Nearly $40 \%$ of 206 had at least one chronic complication related to either Diabetes and/or hypertension. The most common complications reported were peripheral neuropathy, 23(11.2\%), stroke, 21(10.2\%), and chronic kidney disease, $16(7.8 \%)$.

Table 2: Characteristics of 206 diabetic patients with hypertension attending diabetic clinic at $\operatorname{BMC}(\mathrm{n}=206)$.

\begin{tabular}{|c|c|c|}
\hline \multicolumn{2}{|l|}{ Characteristic } & \multirow{2}{*}{$\begin{array}{l}\text { Number \% or Median (IQR) } \\
116(56.3)\end{array}$} \\
\hline Sex & Female & \\
\hline & Male & $90(43.7)$ \\
\hline Age (years) & & 58 (IQR 53-65) \\
\hline \multirow[t]{4}{*}{ Age group (years) } & $<35$ & $8(3.9)$ \\
\hline & $35-50$ & $33(16.0)$ \\
\hline & $51-65$ & $17(56.8)$ \\
\hline & $>65$ & $48(23.3)$ \\
\hline Smoking history & & $23(11.2)$ \\
\hline \multirow[t]{4}{*}{ Systolic blood pressure $(\mathrm{mmHg})$} & $<120$ & $39(18.9)$ \\
\hline & $120-139$ & $56(27.2)$ \\
\hline & $140-159$ & $54(26.2)$ \\
\hline & $\geq 160$ & $57(27.7)$ \\
\hline \multirow[t]{4}{*}{ Diastolic blood pressure (mmHg) } & $<80$ & $43(20.8)$ \\
\hline & $80-89$ & $78(37.9)$ \\
\hline & $90-99$ & $45(21.8)$ \\
\hline & $\geq 100$ & $40(19.4)$ \\
\hline Duration of using anti-hypertensives (Months) & & $48(24-108)$ \\
\hline Duration of DM (Months) & & $65(26-120)$ \\
\hline \multirow[t]{3}{*}{ Average Fasting Blood Glucose (Mmol/dL) } & $\mathrm{FBG}<7$ & $57(27.7)$ \\
\hline & $\mathrm{FBG} \geq 7 \leq 11$ & $69(33.5)$ \\
\hline & $\mathrm{FBG}>11$ & $80(38.3)$ \\
\hline Average total Cholesterol (Mmol/L) & & $4.8(3.8-6.1)$ \\
\hline Patients with documented complications & & $81(39.3)$ \\
\hline \multirow[t]{7}{*}{ Types of complications documented } & Peripheral neuropathy & $23(11.2)$ \\
\hline & Stroke & $21(10.2)$ \\
\hline & Chronic Kidney Disease & $16(7.8)$ \\
\hline & Retinopathy & $13(6.3)$ \\
\hline & Ischemic Heart Disease & $4(1.9)$ \\
\hline & Peripheral Vascular Disease & $2(1.0)$ \\
\hline & Diabetic Foot Ulcer & $1(0.5)$ \\
\hline Using other long term drugs & & $114(59.1)$ \\
\hline \multirow[t]{3}{*}{ Types of drugs used } & Statins & $66(57.9)$ \\
\hline & Aspirin & $35(30.7)$ \\
\hline & Tricyclic anti-depressants & $12(10.52)$ \\
\hline
\end{tabular}

Predictors of hypertension control among diabetic patients with hypertension attending diabetic clinic at BMC: In this study, we found that poor control of hypertension in diabetic patients was significantly associated with poor adherence to anti-hypertensives (OR 1.73[1.262.38 ] $\mathrm{p}=0.002$ ), the presence of any long-term complication (OR 3.19 [1.65-6.18] $\mathrm{p}=0.03$ ) and overweight (BMI>24.9 Vs <24.9) (OR 1.68 [0.982.88], $\mathrm{p}=0.04)$. Furthermore, we have also

DOI: http://dx.doi.org/10.4314/ejhs.v27i5.5 
demonstrated that poor BP control had a positive trend in the patients with the age group of 5665years, and those who were residing within the city with the odds ratio of 2.7 [95\% CI, 1.1-06.9] $(\mathrm{p}=0.04)$ and $3.1[1.1-8.3), \mathrm{p}=0.04)$ respectively in univariate analysis. These features were not significant in multivariate analysis (Table 4).

Prescription pattern of anti-hypertensives among diabetic patients: All, except one patient, were prescribed on at least one antihypertensive drug and among them 105 (51.2\%), 80 (39.0\%) and $9(4.4 \%)$ were on monotherapy, two drugs combination and three/more drugs combination respectively. Median duration on antihypertensives was 48 months (IQR 24-108). Types of anti-hypertensives used were Angiotensin II converting enzyme Inhibitors (ACEI) $112(54.6 \%)$ and angiotensin II receptor blocker (ARB) $62(30.2 \%)$, Calcium channel blockers (CCB) 92 (44.9\%), Beta-Blockers (BB) 8 (3.9\%), Diuretics 7 (3.4\%) and Hydralazine 6 (2.9\%). Details of anti-hypertensives prescription pattern have been shown in Table 3.

Table 3: Prescription patterns of anti-hypertensives among 205 diabetic patients

\begin{tabular}{lllllll}
\hline Drug & $\begin{array}{l}\text { Total (\%) } \\
\text { n=205 }\end{array}$ & $\begin{array}{l}\text { Monotherapy } \\
\mathbf{1 0 5}(\mathbf{5 1 . 2 \%})\end{array}$ & Two drugs 80 (39.0\%) & Three or more drugs 9 (4.4\%) \\
& & & & & & \\
ACEI & $107(52.2)$ & $60(57.1 \%)$ & ACEI \& CCB & $35(43.8)$ & BB, CCB \& Hydralazine & $3(33.3)$ \\
CCB & $92(44.9)$ & $19(18.1)$ & ARB \& CCB & $22(27.5)$ & ARB, CCB\& Diuretics & $2(22.2)$ \\
ARB & $51(24.9)$ & $17(16.2 \%)$ & ACEI \& ARB & $8(10.0)$ & ACEI, CCB \& Hydralazine & $2(22.2)$ \\
BB & $10(4.9)$ & 0 & BB \& CCB & $5(6.3)$ & BB, Hydralazine \& CCB & $1(11.1)$ \\
Diuretics & $8(3.9)$ & 0 & CCB \& Diuretics & $3(3.8)$ & ACEI, ARB \& BB & $1(11.1)$ \\
Hydralazine & $6(2.9)$ & 0 & ARB \& Diuretics & $1(1.3)$ & & \\
& & & ACEI \& Diuretics & $1(1.3)$ & & \\
\hline
\end{tabular}

\section{DISCUSSION}

In this study, we found that nearly $70 \%$ of diabetic patients had hypertension and only few (15.5\%) reached their target BP values. Poor adherence to anti-hypertensive medications, the presence of any long-term complication and overweight/obesity significantly predicted poor control of hypertension in these patients.

Trends of data collected by the World Health Organization (WHO) suggest that the number of people with uncontrolled hypertension has risen over the recent years, and the highest being in Africa (30\%). The prevalence of diabetes has also been increasing globally, particularly in middle and lower income countries (14). These trends undoubtedly reflect the reported high magnitude of co-morbidity between the two diseases (11-13). In Tanzania, Mwita et al reported the prevalence of hypertension among diabetic patients to be $55 \%$ at Muhimbili National Hospital in 2012(7), where lower prevalence of $29.3 \%$ was reported at the same center two decades ago (18). In the index study, we have demonstrated this magnitude to be much higher $(69.8 \%)$. This drift clearly suggests a radical increase of this burden in the country.

We have observed a low rate $(15.5 \%)$ of control of hypertension in the index study. Comparably similar rates have been observed in Tanzania (34\%), Cameroun (10.2\%) and Malaysia $(23.5 \%)(7,15,19)$. Several randomized trials have associated significant clinical benefits of tight BP control among diabetic patients (20-22). In the United Kingdom Prospective Diabetes Study (UKPDS), for instance, it was found that each $10 \mathrm{mmHg}$ reduction in systolic BP was associated with average reduction of $15 \%$ in diabetes-related mortalities due to myocardial infarction (11\%) and micro vascular complications (retinopathy and nephropathy) (13\%) (20). Nearly $40 \%$ of our patients already had at least one chronic complication due to Diabetes/ hypertension/both. 
Table 4: Logistic regression analysis for predictors of poor control of blood pressure among diabetic patients with hypertension attending diabetic clinic at BMC

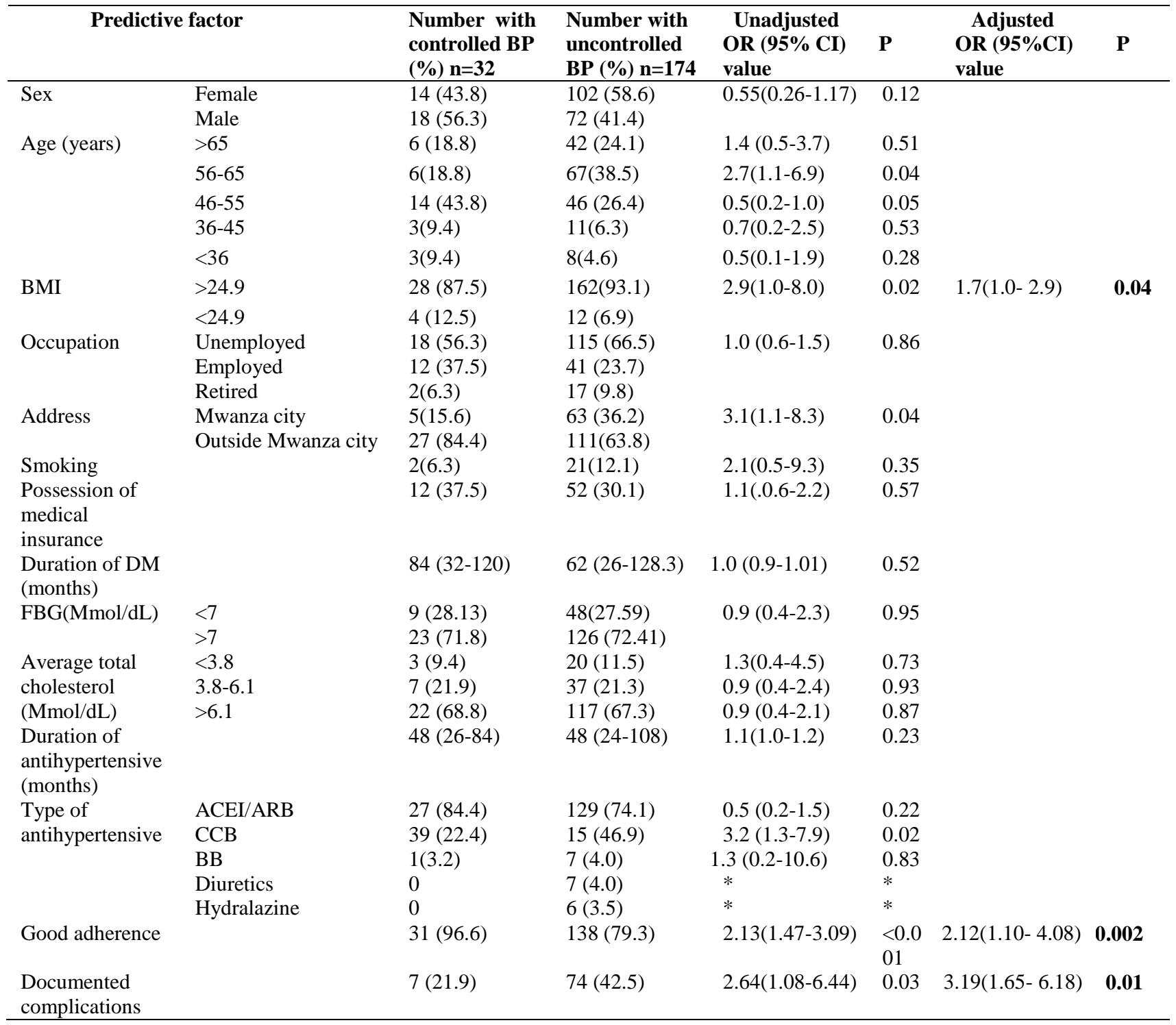

Good adherence to anti-hypertensive medications among diabetic patients has been associated with better hypertension control (23) and decreased risk of CV disease, CV events and mortality (24). Similar to our findings, adherence to antihypertensives among diabetic patients is generally poor $(25,26)$. This might be partly caused by failure of the patients to prevail with increased pile of drugs to be taken, or potentiated side effects from both anti-hypertensives and hypoglycemics.
In the case study, we have observed a direct association between overweight/obesity and poor control of BP. Similarly, a large Swedish survey of National Diabetes register which recruited more than 1500 patients demonstrated the same findings. In this survey, both lower BMI at baseline and during follow-up had a favorable association for control of BP (27). Chew et al reported similar findings in Malaysia (19). A combination of Diabetes, hypertension and obesity has been

DOI: http://dx.doi.org/10.4314/ejhs.v27i5.5 
related with autonomic imbalance and circadian disruption of the autonomic tones during day and night periods. These effects may contribute to the observed findings (28).

Most patients with diabetes require multiple anti-hypertensive medications to achieve the target blood pressure. (20,29). A meticulous combination of anti-hypertensive is therefore desirable. Underprescription of anti-hypertensives was observed in this study where less than one-half $(43.4 \%)$ of our patients were prescribed on two or more antihypertensives. Despite that short-coming, the majority of our patients were prescribed on ReninAngiotensin system blockers either in combination with other agents or as a monotherapy. This prescription pattern is in agreement with several international guidelines due to their role in significant reduction of CVD-related deaths, stroke and delaying deterioration of Glomerular Filtration Rate (GFR) in patients with Chronic Kidney Disease (CKD) (8,10,30,31). Unexpectedly, ACEI with ARB was prescribed in nine patients. This regime is not recommended as it does not have any added advantage (32). Human errors might be the reasons for these incorrect prescriptions.

In conclusion, the prevalence of poor control of hypertension among diabetic patients in a tertiary hospital is high and it is highly predicted by modifiable factors. Incongruous prescription of anti-hypertensives is also evident. We believe that our findings will encourage health care providers to deliver the health services according to guidelines and the patient's individualization. Importantly, clinicians should endlessly continue to provide health education on adherence, preventive measures and control of NCDs. Further follow-up studies are necessary to ascertain the long-term outcome of these patients.

This study has limitations. Being a cross sectional study, the fate of diabetes and hypertension co-morbidity could not be assessed. The time from diagnosis of diabetes and hypertension could also not be evaluated. Despite these shortcomings, we believe that these findings are important and relevant as they provide a broad picture of the situation locally and a baseline information for further studies on the subject.

\section{ACKNOWLEDGEMENTS}

The authors are thankful to the director general of Bugando Medical Center and staff members in the diabetic clinic and medical records of Bugando Medical Center.

\section{REFERENCES}

1. World Bank. Human Development Network. The growing danger of non-communicable diseases. Acting now to reverse course 2011. http://wwwds.worldbank.org/external/default/WDSContentSer ver/WDSP/IB/2011/11/30/000386194_201111300 23857/Rendered/PDF/657850WP0Box360C00WB DeepeningCrisis.pdf

2. Dalal S, Beunza JJ, Volmink J, et al. Noncommunicable diseases in sub-Saharan Africa: what we know now. Int $J$ Epidemiol 2011; 40(4):885-901.

3. World Health Organization. The health of the people. What works 2013. http://www.who.int/bulletin/africanhealth2014/dise ase_threats/en/

4. World Health Organization. Health statistics and information 2012. http://www.who.int/healthinfo/global_burden_dise ase/estimates/en/index1.html

5. 5. Kahya EN, Harman E, Dolek D, et al. Rate of blood pressure control and antihypertensive treatment approaches in diabetic patients with hypertension. Turk Kardiyol Dern Ars 2014; 42(8):733-40.

6. Jansson SP, Andersson DK, Svardsudd K. Mortality Trends in Subjects With and Without Diabetes During 33 Years of Follow-up. Diabetes Care 2009; 33(3):551-6.

7. Mwita JC, Mugusi F, Lwakatare J, et al. Hypertension control and other cardiovascular risk factors among diabetic patients at Muhimbili National Hospital, Tanzania. East Afr J Public Health 2012; 9(2):70-3.

8. Fox CS, Golden SH, Anderson C, et al. Update on Prevention of Cardiovascular Disease in Adults With Type 2 Diabetes Mellitus in Light of Recent Evidence: A Scientific Statement From the American Heart Association and the American Diabetes Association. Diabetes Care 2015; 38(9):dci150012.

9. Chen G, McAlister FA, Walker RL, et al. Cardiovascular outcomes in framingham participants with diabetes: the importance of blood pressure. Hypertension 2011; 57(5):891-7.

DOI: http://dx.doi.org/10.4314/ejhs.v27i5.5 
10. Chobanian AV, Bakris GL, Black HR, et al. Seventh report of the Joint National Committee on Prevention, Detection, Evaluation, and Treatment of High Blood Pressure. Hypertension 2003; 42(6):1206-52.

11. Chew BH, Mastura I, Shariff GS, et al. Determinants of uncontrolled hypertension in adult type 2 diabetes mellitus: an analysis of the Malaysian diabetes registry 2009. Cardiovasc Diabetol 2012; 11:54.

12. Rwegerera GM. Adherence to anti-diabetic drugs among patients with Type 2 diabetes mellitus at Muhimbili National Hospital, Dar es Salaam, Tanzania- A cross-sectional study. Pan Afr Med J 2014;17:252.

13. Wei $\mathrm{N}$, Zheng $\mathrm{H}$, Nathan DM. Empirically establishing blood glucose targets to achieve HbA1c goals. Diabetes Care 2014; 37(4):1048.

14. World Health Organization. Global status report on noncommunicable diseases 2014. http://apps.who.int/iris/bitstream/10665/148114/1/ 9789241564854_eng.pdf?ua=1

15. Choukem SP, Kengne AP, Dehayem YM, et al. Hypertension in people with diabetes in subSaharan Africa: revealing the hidden face of the iceberg. Diabetes Res Clin Pract 2007; 77(2):293.

16. Lee HS, Lee SS, Hwang IY, et al. Prevalence, awareness, treatment and control of hypertension in adults with diagnosed diabetes: the Fourth Korea National Health and Nutrition Examination Survey (KNHANES IV). J Hum Hypertens 2013; 27(6):381-7.

17. Mohan V, Seedat YK, Pradeepa R. The rising burden of diabetes and hypertension in southeast asian and african regions: need for effective strategies for prevention and control in primary health care settings. Int J Hypertens 2013;

18. Mugusi F, Ramaiya KL, Chale S, et al. Blood pressure changes in diabetes in urban Tanzania. Acta Diabetol 1995; 32(1):28-31.

19. Chew BH, Mastura I, Shariff GS, et al. Determinants of uncontrolled hypertension in adult type 2 diabetes mellitus: an analysis of the Malaysian diabetes registry 2009. Cardiovasc Diabetol; 11:54.

20. Turner R, Holman R, Stratton I, et al. Tight blood pressure control and risk of macrovascular and microvascular complications in type 2 diabetes: UKPDS 38. UK Prospective Diabetes Study Group. BMJ 1998; 317(7160):703-13.

21. Kaplan NM. Vascular outcome in type 2 diabetes: an ADVANCE? Lancet 2007; 370(9590):804-5.
22. Hansson L, Zanchetti A, Carruthers SG, et al. Effects of intensive blood-pressure lowering and low-dose aspirin in patients with hypertension: principal results of the Hypertension Optimal Treatment (HOT) randomised trial. HOT Study Group. Lancet 1998; 351(9118):1755-62.

23. Putnam W, Lawson B, Buhariwalla F, et al. Hypertension and type 2 diabetes: what family physicians can do to improve control of blood pressure-an observational study. BMC Fam Pract 2011; $12: 86$.

24. Degli EL, Saragoni S, Benemei S, et al. Adherence to antihypertensive medications and health outcomes among newly treated hypertensive patients. Clinicoecon Outcomes Res 2011; 3:47.

25. Kivimäki M, Batty GD, Hamer M, et al. Influence of retirement on nonadherence to medication for hypertension and diabetes. CMAJ 2013; 185(17):E784-90.

26. Sansbury B, Dasgupta A, Guthrie L, et al. Time perspective and medication adherence among individuals with hypertension or diabetes mellitus. Patient Educ Couns 2014; 95(1):104-10.

27. Nilsson PM, Cederholm J, Gudbjörnsdottir S, et al. Predictors of successful long-term blood pressure control in type 2 diabetic patients: data from the Swedish National Diabetes Register (NDR). J Hypertens 2005; 23(12):2305-11.

28. Boer ML, Figueiredo VN, Demacq C, et al. Relationship of autonomic imbalance and circadian disruption with obesity and type 2 diabetes in resistant hypertensive patients. Cardiovasc Diabetol 2011; 10:24.

29. Bakris GL. A practical approach to achieving recommended blood pressure goals in diabetic patients. Arch Intern Med;161(22):2661.

30. Remuzzi G, Schieppati A, Ruggenenti P. Clinical practice. Nephropathy in patients with type 2 diabetes. N Engl J Med. 2002; 346(15):1145-51.

31. Effects of ramipril on cardiovascular and microvascular outcomes in people with diabetes mellitus: results of the HOPE study and MICROHOPE substudy. Heart Outcomes Prevention Evaluation Study Investigators. Lancet 2000; 355(9200):253-9.

32. Mancia G, Fagard R, Narkiewicz K, et al. 2013 ESH/ESC guidelines for the management of arterial hypertension. Blood press 2014; 23(1):316. 2013. 